\title{
Podcasting Computer Science E-1
}

\section{Citation}

Malan, David J. 2007. Podcasting Computer Science E-1. In SIGCSE 2007: Proceedings of the Thirty-Eighth SIGCSE Technical Symposium on Computer Science Education, March 7-10, 2007, Covington, Kentucky, ed. ACM SIGCSE and Ingrid Russell, 389-393. New York, N.Y.: Association for Computing Machinery.

\section{Published Version}

http://dx.doi.org/10.1145/1227310.1227446

\section{Permanent link}

http://nrs.harvard.edu/urn-3:HUL.InstRepos:2829931

\section{Terms of Use}

This article was downloaded from Harvard University's DASH repository, and is made available under the terms and conditions applicable to Other Posted Material, as set forth at http:// nrs.harvard.edu/urn-3:HUL.InstRepos:dash.current.terms-of-use\#LAA

\section{Share Your Story}

The Harvard community has made this article openly available.

Please share how this access benefits you. Submit a story.

\section{Accessibility}




\title{
Podcasting Computer Science E-1
}

\author{
David J. Malan \\ Division of Engineering and Applied Sciences \\ Harvard University \\ Cambridge, Massachusetts, USA \\ malan@post.harvard.edu
}

\begin{abstract}
In recent months have teachers become publishers of content and students subscribers thereof by way of podcasts, feeds of audio, video, and other content that can be downloaded to clients like iTunes and devices like iPods. In the fall of 2005, we ourselves began to podcast Harvard Extension School's Computer Science E-1 in both audio and video formats, the first course within Harvard University to do so. Our goals were to provide students with more portable access to educational content and to involve them in technology itself.

To evaluate this experiment, we have analyzed logs and surveys of students. We find that our students valued E-1's podcast more as a vehicle for review (45\%) than as an alternative to attendance $(18 \%)$. We also find that most students $(71 \%)$ tended to listen to or watch lectures on their computers, with far fewer relying upon audio-only (19\%) or video (10\%) iPods. We argue, meanwhile, that podcasting, despite its widespread popularity, is but a marginal improvement on trends long in progress. It is this technology's reach that we claim is significant, not the technology itself. Logs suggest that E-1's own podcast, available not only to students but to the public at large, has acquired (as of September 2006) between 6,000 and 10,000 subscribers from over 50 countries. We argue, then, that podcasting offers to extend universities' educational reach more than it offers to improve education itself.
\end{abstract}

\section{Categories and Subject Descriptors}

K.3.1 [COMPUTERS AND EDUCATION]: Computer Uses in Education-Distance learning; K.3.2 [COMPUTERS AND EDUCATION]: Computer and Information Science Education-Computer science education

\section{General Terms}

Design, Experimentation, Human Factors

\section{Keywords}

distance education, iPod, iTunes, podcast, podcasting

Permission to make digital or hard copies of all or part of this work for personal or classroom use is granted without fee provided that copies are not made or distributed for profit or commercial advantage and that copies bear this notice and the full citation on the first page. To copy otherwise, to republish, to post on servers or to redistribute to lists, requires prior specific permission and/or a fee.

SIGCSE'07, March 7-10, 2007, Covington, Kentucky, USA.

Copyright 2007 ACM 1-59593-361-1/07/0003 ...\$5.00.

\section{INTRODUCTION}

In the limelight of late is podcasting: distribution of audio, video, and other content via feeds to which one can subscribe with clients like iTunes [4]. Otherwise known as podcasts, these feeds enable publishers to push new content to a subscriber's computer or mobile device (e.g., iPod [3]) for subsequent playback offline.

In recent months have teachers become publishers and students subscribers, with lectures themselves the content. We ourselves set out in the fall term of 2005 to podcast Computer Science E-1: Understanding Computers and the Internet, an introductory course at Harvard Extension School [6] that, per its syllabus, "[demystifies] computers and the Internet, along with their jargon, so that students understand not only what they can do with each but also how it all works and why." The first course within Harvard University to be podcasted in both audio and video formats, E-1's lectures were made available not only to registered students but also to the public at large (at no charge), along with some sections (i.e., recitations) and workshops led by the course's teaching fellows. Our goals were to provide our own students with more portable means of accessing the course's content and to involve them with technology that the course itself explores.

Not only did we gather statistics on downloads throughout this experiment, we also surveyed our students for their thoughts on the technology. We present in this paper the results of our trial along with details of its implementation. Along the way, we argue that the value of podcasting in students' education has been overstated with hype, noting instead that this delivery mechanism is but a marginal improvement on trends already in progress. We view podcasts of courses as most valuable for those not even enrolled. In that podcasts and directories thereof centralize and facilitate discovery of educational content, podcasting offers universities new opportunities to teach, sans credits and degrees, those for whom attendance, enrollment, or education itself might not otherwise be possible.

We find that our own students valued E-1's podcast more as a vehicle for review (45\%) than as an alternative to attendance (18\%). We also find that most of our students $(71 \%)$ tended to listen to or watch lectures on their computers, rather than on audio-only (19\%) or video (10\%) iPods. Perhaps most striking, though, is the size of the course's unofficial student body: logs suggest that the podcast has acquired (as of September 2006) between 6,000 and 10,000 subscribers, just over half $(62 \%)$ from the United States and the rest from over 50 other countries. We offer these results 


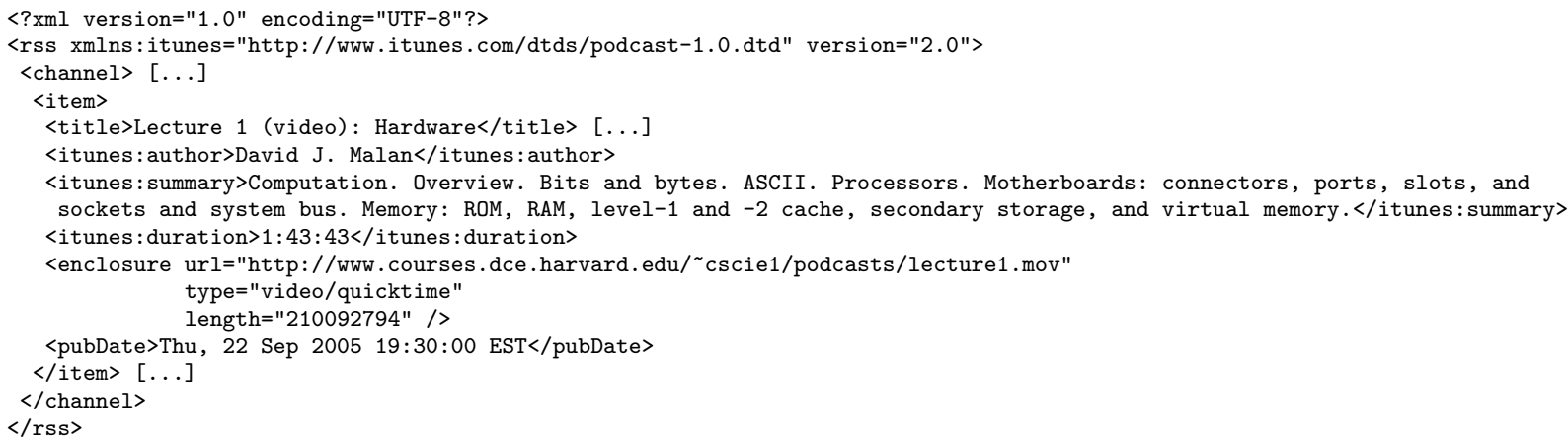

Figure 1: E-1's podcast is defined by its RSS (i.e., XML) feed, a text file that describes (and provides URLs to) the podcast's actual content. iTunes (like other clients) monitors this file for changes and relies upon it to generate its interface (Figure 2). Students subscribe to the podcast by subscribing to this feed's URL. This excerpt describes Lecture 1's video.

as evidence of podcasting's potential not necessarily to educate better but to educate further.

In the two sections that follow, we elaborate on E-1 and the implementation of its podcast. In Section 4, we present the results of our trial. In Section 5, we offer discussion thereof. In Section 6, we introduce work that has resulted from this experience. In Section 7, we conclude.

\section{ABOUT E-1}

A gateway to other courses in computer science, E-1 exposes students to material in breadth through lectures on hardware, software, the Internet, multimedia, security, website development, programming, and more. Though some students take the course in pursuit of certificates or degrees at Harvard Extension School, many enroll for their own edification and continuing education. Though the course does offer hands-on sections and workshops, the course's lectures are more conceptual than practical. Per its syllabus, "This course is all about understanding: understanding what's going on inside your computer when you flip on the switch, why tech support has you constantly rebooting your computer, how everything you do on the Internet can be watched by others, and how your computer can become infected with a worm just by turning it on. . . . Students leave this course armed with a new vocabulary and equipped for further exploration of computers and the Internet."

Since 1999 has E-1 been part of Harvard Extension School's Distance Education [7] program, whereby students can take the course via the Internet. Lectures are filmed, digitized, and posted online in RealAudio [9] and RealVideo [10] formats, both of which are synchronized with HTML-based slideshows of lectures' slides.

Enrollment each fall hovers around 60 students, up to a third of whom are typically distant from Cambridge. The URL of E-1's website appears in this paper's appendix.

\section{PODCASTING E-1}

To expedite production of the course's podcast each week, we simply converted our RealAudio and RealVideo formats to MP3 [8] and QuickTime [5] formats, respectively. Through trial and error, we found acceptable middle ground between files' quality and size: we encoded MP3s in monophone at a sample rate of 22 kilohertz and at a bitrate of 56 kilobits per second; we encoded QuickTime movies with an MPEG4 codec at 30 frames per second, at a resolution of $320 \times 240$ pixels, and at a bitrate of 240 kilobits per second. For each two-hour lecture, the corresponding MP3 was about 40 megabytes, while the corresponding QuickTime movie was about 200 megabytes.

We then uploaded these files (along with PDFs of slides) each week to a server on campus and updated the podcast's RSS (i.e., XML) feed (Figure 1), a text file that describes (and provides URLs to) the podcast's actual content. ${ }^{*}$ To "subscribe" to E-1's podcast, then, is to monitor this feed's URL for changes with a client like iTunes, which generates its interface (Figure 2) based on the feed's metadata.

In this manner was each lecture made available to students via iTunes and other clients, usually within 48 hours of its delivery on campus. The URL of E-1's podcast (i.e., its RSS feed) appears in this paper's appendix.

\section{RESULTS}

To evaluate our trial, we turned to logs and students themselves. Though our own students' thoughts on the podcast proved quite positive, we were struck more by the public's response. We present both in turn.

\subsection{Students' Response}

At term's end, we presented students with surveys about E-1's podcast. Though students - local and distant alikealso had access to the course's lectures in RealAudio and RealVideo formats, these surveys made clear that questions only regarded the MP3- and QuickTime-based podcast rather than distance education itself.

Among the 22 respondents, all but one had utilized the course's podcast. The predominant reason was not to avoid attendance but, rather, to review lectures already attended: $45 \%$ valued the podcast as a vehicle for review, whereas $18 \%$ valued it as an alternative to attendance. Nonetheless, we did not detect an $18 \%$ (or even noticeable) drop in attendance at lectures vis-à-vis prior semesters, presumably because the course already offered distance education.

Nonetheless, some students did express a preference for the course's RealVideo format over the podcast's QuickTime format, the former of which offered slightly higher resolution as well as synchronized slides. Other students preferred the podcast's portability. One student explained: "I love this feature, I have an hour to an hour and a half commute each

\footnotetext{
* To accommodate the visually impaired, we also offered hyperlinks to the podcast's content on the course's website, whose text is more easily recited than iTunes's by accessibility software.
} 


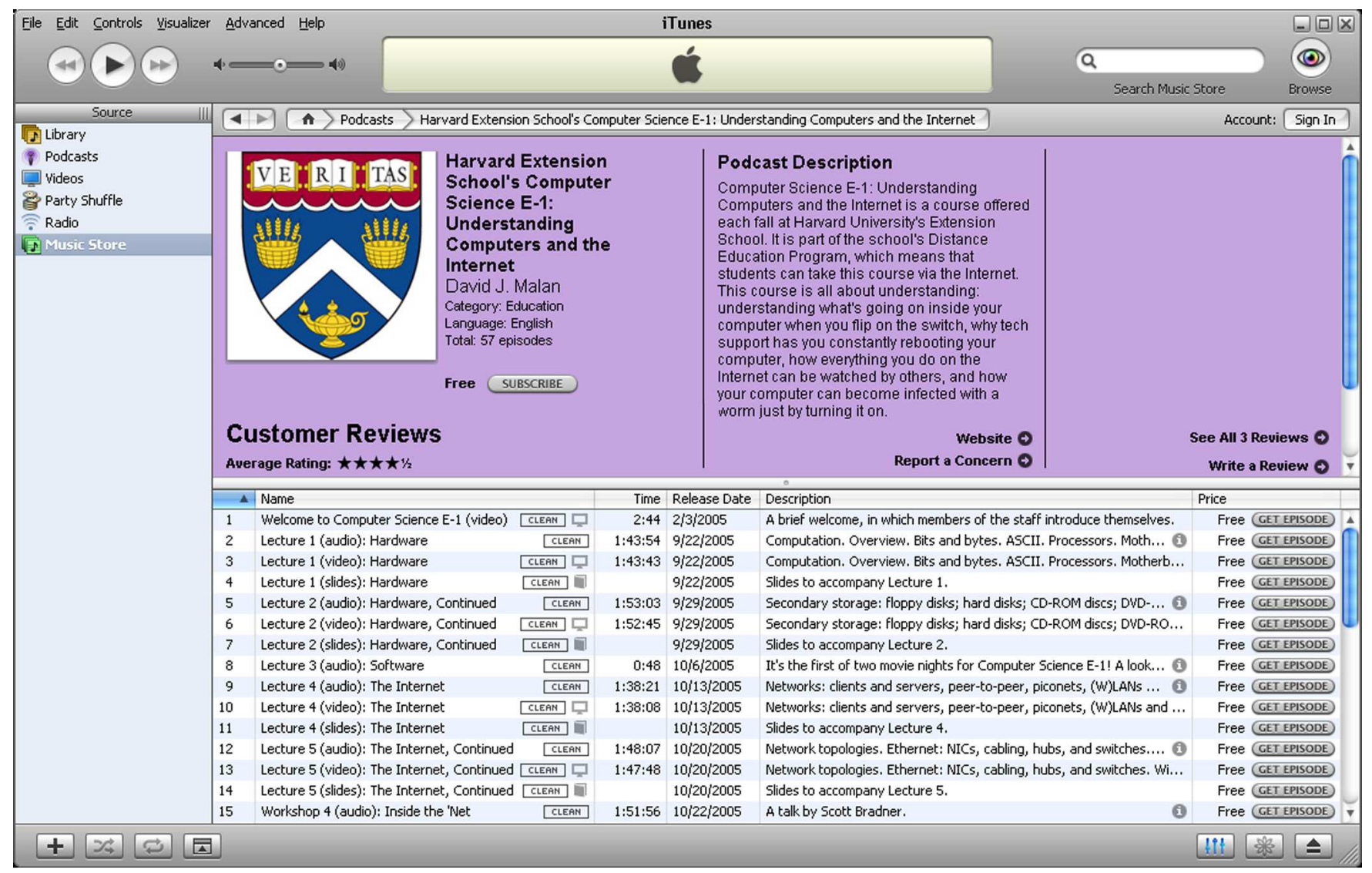

Figure 2: E-1's podcast was made available to students and the public at large via iTunes and other podcast directories. Included in the podcast were MP3s and QuickTime movies of lectures (along with PDFs of slides). Also included were some sections and workshops led by the teaching fellows. Pictured is the interface generated by iTunes based on the podcast's RSS feed (Figure 1).

way to work everyday and to have the podcasts helped with reviewing the lectures (on the go)."

We also surveyed students on the means by which they tended to listen to or watch the podcast's content. Most students $(71 \%)$ tended to listen to or watch lectures on their computers, rather than on audio-only (19\%) or video (10\%) iPods (Figure 3). Though worthy of note, this distribution is not wholly surprising. After all, Harvard Extension School's student body is more diverse in age than typical college classrooms, whose students might be more likely than ours to own the latest technology. Moreover, E-1's students are perhaps, by definition, less technically savvy than those who tend to own the same. And, in fairness, the video iPod was only released toward the start of our trial.

However, this preference for computers over iPods might also reflect disparity between students' attitudes toward classes on iPods and actual usage thereof. Our own students' comments suggest as much: "I really liked [the] concept of using podcasting as an educational medium. It extends the reach in very affordable ways. I think I only listened to one podcast and none of the videos." In the words of another, "I have not used [podcasting] once this semester, either for video or for audio. Though, I absolutely like that the opportunity to utilize these components exists." The sentiment was not uncommon: "I think podcasting is a good idea. The more formats information is made available in, the better. However, I did not listen to any of the podcasts."
To be sure, sheer novelty explains some students' enthusiasm for the podcast: "I think it was also very much in line with the course curriculum to have students learn about and use the technologies that are available, including the iPod. How very cutting edge we were!" But some students' takes on the podcast were more matters of fact: "It's simply a good way to review and listen to lectures."

Needless to say, our own students' response was ultimately positive. But the public response was more striking.

\subsection{Public Response}

In order to make E-1's podcast available not only to our own students but also the public at large, we submitted the URL of its RSS feed to iTunes's podcast directory at term's start as well as to other web-based directories. Thanks, in part, to those directories as well as to several articles and blogs that brought attention to the course's podcast upon its release, the sizes of E-1's official and unofficial student bodies ultimately differed by orders of magnitude.

Logs suggest that the podcast has garnered (as of September 2006) between 6,000 and 10,000 subscribers (Figure 4). Equally striking is the podcast's reach. Logs suggest that just over half $(62 \%)$ of subscribers were from the United States, with other subscribers scattered across more than 50 other countries (Figure 5).

Even more surprising than these numbers alone are the dozens of emails that we have received from subscribers. 


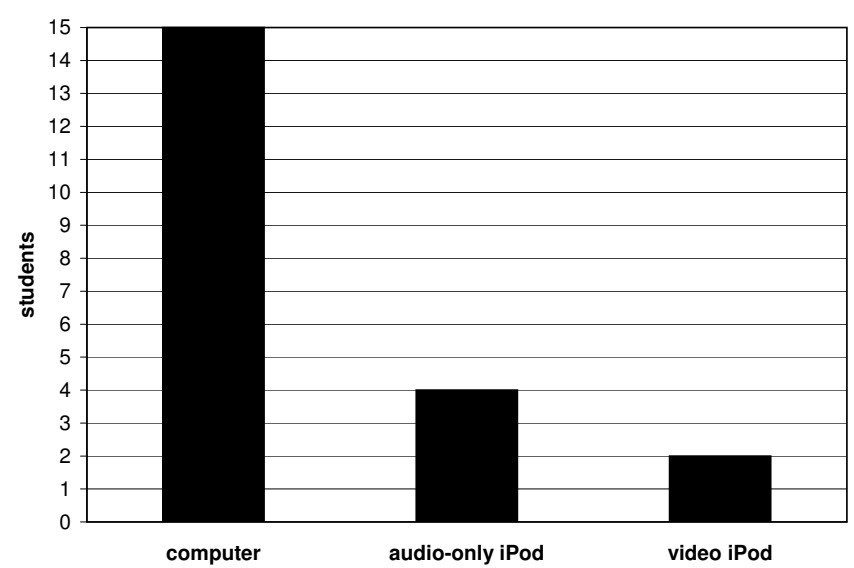

Figure 3: At term's end, we surveyed students on how they listened to or watched the course's podcast. Among the 21 respondents, $15(71 \%)$ tended to listen or watch on their computers, $4(19 \%)$ tended to listen on audioonly iPods, and $2(10 \%)$ tended to watch on video iPods.

Many have inquired as to how to enroll in E-1 or other courses at a distance, evidence that the course's podcast has reached corners of the country and world that the school's catalogue has not. One subscriber writes: "I am from Vienna, Austria and how can I register [for your] distance education program? I'm podcasting your course and . . . [hope to] be a legit [distance-education] student in your class, thanks a lot for creating [distance education]."

Other subscribers appreciate that the podcast's content has reached them at all: "I live in the central interior of British Columbia, Canada, and am surrounded by forest, the nearest town is twenty minutes drive. . . . I pay a great deal of money to connect to the Internet via satellite. . . . I consider the lessons I have learned from you and your colleagues to have completely paid for this service." Another subscriber writes: "I would like to thank you for posting your Comp. Sci. E-1 lectures at Harvard University as podcasts. The commute to Cambridge from the Upper Galilee in Israel is a bit too much for me to attend the course in person. Nevertheless, your podcasts have been a great 'second best."'

Among those who have emailed us are also other educators, many of whom find the podcast's content and their own materials complementary: "My International Baccalaureate students here . . . in Penzance, UK have all subscribed and consider it to be an essential tool in their learning already."

Meanwhile, other subscribers view the podcast not only as a class but as an opportunity: "I trust that you won't mind me saying that we now have the benefit of being tutored by one of the world's foremost academic institutions from right here in South Africa." In the words of another subscriber, "It is not everyday that someone can be let into the halls of Harvard U and experience the richness of an Ivy League experience." Other emails embody, quite simply, gratitude: "Thanks again to you and Harvard for providing the podcasts."

To be sure, a podcast about computers and the Internet perhaps cannot help but be popular among those already interested in computers and the Internet (i.e., E-1's subscribers). That over 6,000, though, took an interest in just one course's podcast does seem to confirm the technology's power to extend schools' reach well beyond their walls, ivy-

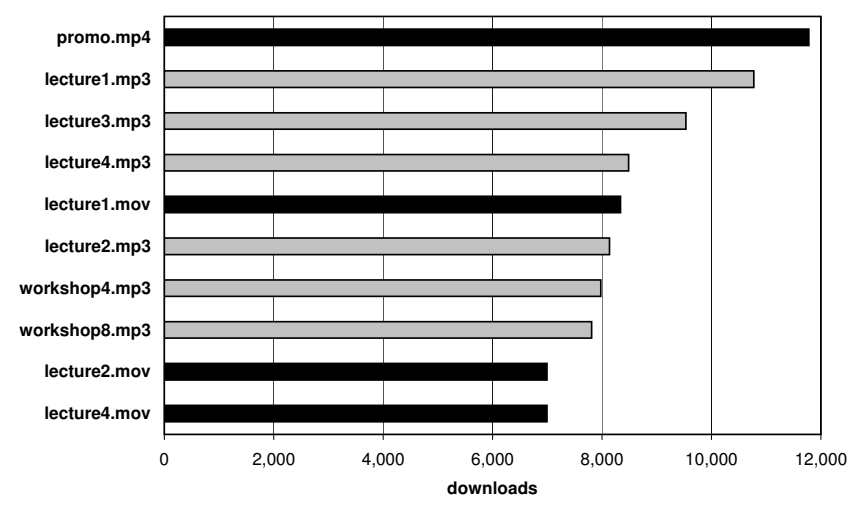

Figure 4: Logs suggest that E-1's podcast has had between 6,000 and 10,000 subscribers. Presented are some of the podcast's most popular downloads, with video content colored in black and audio content colored in gray.

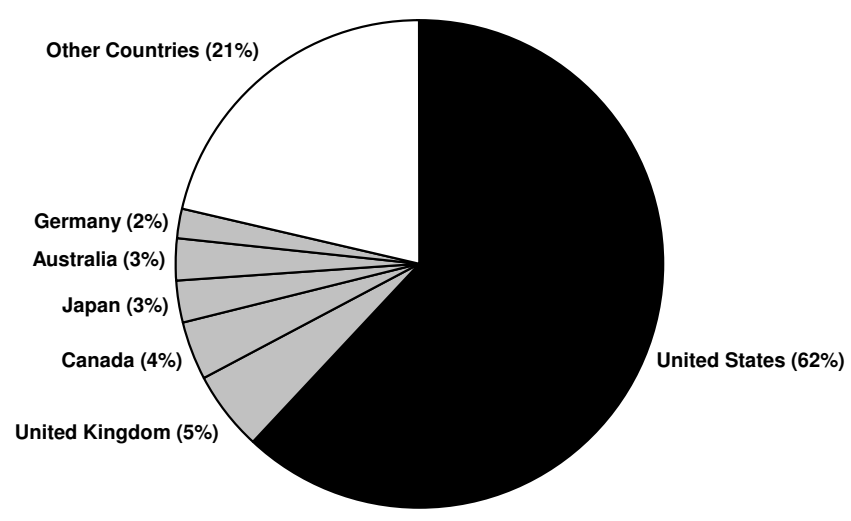

Figure 5: Logs suggest that just over half (62\%) of the subscribers to E-1's podcast reside in the United States, with other subscribers scattered across more than 50 other countries.

covered or not. Moreover, that so many subscribers engaged in a course without promise of credit or degree suggests that podcasting offers potential not necessarily to educate better but to educate further. We explore these ideas further in the section that follows.

\section{DISCUSSION}

Podcasting may be new, but cameras in classrooms are not. For years have universities filmed some courses' lectures so that students can watch recordings thereof if absent from class or in need of review. What podcasting ultimately offers are marginal improvements on lectures' storage and delivery.

The earliest of media for recordings of lectures (e.g., VHS) offered limited bandwidth: only one student could borrow a tape at a time. Even before podcasting's advent had digital storage and delivery of lectures already begun to supplant magnetic, thanks, in part, to faster networks and processors, better codecs, and cheaper storage. As early as 1998 was Harvard College, for instance, streaming some lectures across campus via Microsoft NetShow. With digital storage and delivery, not only can multiple students now "borrow" the same content at once, they can usually watch it anywhere they have Internet access. Distance education has further popularized the medium, with videos now commonly presented alongside other multimedia. 
In that it offers offline as opposed to streaming playback, then, podcasting has simply freed students of the "tethers" of wired and even wireless Internet access. We daresay that podcasting's potential for academia has been overstated with hype: "Could ivy-covered lecture halls become as obsolete as the typewriter?" [11] Not likely, we argue. The educational value of podcasting as a tool for review seems clear. The educational value of podcasting as a replacement for live lectures, we admit, depends on the student, as the medium might very well be perfect for some students' styles of learning. But on the whole, we view classes on iTunes or iPods as "the next best thing" for students for whom attendance or enrollment is not otherwise possible. What iTunes and similar podcast directories offer is ease of access to educational content. Like any good software, they lower a bar. Insofar as podcasting centralizes and facilitates discovery of educational content, it offers universities new opportunities to teach, sans credits and degrees, populations at large. By no means are all courses suited for the medium. But even a few dozen courses from a few dozen universities could still fill a podcast directory.

\section{FUTURE WORK}

We plan to podcast E-1 in the fall of 2006 as well. Based on feedback from students and subscribers, though, we plan to offer additional content. Striking among 2005's logs is the popularity of two of E-1's workshops (Figure 4). Whereas E-1's lectures explore broad categories of topics (e.g., hardware), workshops emphasize individual topics (e.g., digital photography). The popularity of the latter suggests to us an interest in more targeted content. Comments from students on surveys seem to confirm: "I would like the material to be in [different] lengths: a two-minute "you really [have] to know this' . . . and lectures." Another student offers: "Perhaps you should consider including educational podcasts not just teaching podcasts."

Not only, then, will we continue to podcast lectures, sections, and workshops, we also plan to include "videos of the week," short segments produced by the teaching fellows on individual topics relevant to that week's lecture. Whereas lectures will remain comprehensive, these "bite-sized" videos will instead "play to the camera," offering students more focused knowledge on demand.

Moreover, this content will be made available not only via podcast but also via Flash [2]. With Flash reportedly installed on $97.3 \%$ of Internet-enabled desktops [1], we intend for this medium to lower the bar to E-1's content even further, particularly for visitors without iTunes or iPods (or even knowledge thereof).

\section{CONCLUSION}

In the fall of 2005, we began to podcast Harvard Extension School's Computer Science E-1 in both audio and video formats, the first course within Harvard University to do so. Our goals were to provide our own students with more portable access to the course's content and to involve them in technology itself.

Through surveys at term's end, we have found that students valued the podcast more as a vehicle for review (45\%) than as an alternative to attendance (18\%). We have also found that most students $(71 \%)$ tended to listen to or watch lectures on their computers, with far fewer relying upon audio-only (19\%) or video (10\%) iPods. Most striking, however, is the podcast's reach. Logs suggest that the podcast has acquired between 6,000 and 10,000 subscribers from over 50 countries.

Despite the podcast's popularity, we have argued that podcasting, as a delivery mechanism, is but a marginal improvement on trends long in progress. Moreover, we have argued that podcasting offers to extend universities' educational reach more than it offers to improve education itself.

Not only do we plan to podcast E-1 again in the fall of 2006, we also plan to experiment with alternative forms of content so as to revisit this work's questions with additional data and experience.

\section{APPENDIX}

The URL of E-1's website is

http: //www. fas.harvard.edu/ cscie1/.

The URL of E-1's podcast (i.e., its RSS feed) is http://www.fas.harvard.edu/ cscie1/podcast/.

\section{ACKNOWLEDGEMENTS}

Many thanks to E-1's teaching fellows-Rei Diaz, Dan Armendariz, and Roman Rubinstein - without whose time and effort this trial would not have been possible. Many thanks as well to Elizabeth Hess, Mary Higgins, Chris Mehl, Rod Lindheim, and Michael Shinagel for their support of this experiment as well as to Michael D. Smith for his assistance with this work. Many thanks to E-1's students and subscribers, without whose interest and thoughts this work would not have been possible. And heartfelt thanks to Henry H. Leitner for all this and more.

\section{REFERENCES}

[1] Adobe Systems Incorporated. Flash Player Statistics. http://www . adobe.com/products/player_census/ flashplayer/.

[2] Adobe Systems Incorporated. Flash Professional 8. http://www .adobe.com/products/flash/flashpro/.

[3] Apple Computer, Inc. iPod Family. http://www . apple.com/ipod/.

[4] Apple Computer, Inc. iTunes Overview. http://www .apple.com/itunes/overview/.

[5] Apple Computer, Inc. QuickTime. http://www .apple.com/quicktime/.

[6] Harvard Extension School. http://www. extension.harvard.edu/.

[7] Harvard Extension School. Distance Education. http://www . extension.harvard.edu/DistanceEd/.

[8] Moving Picture Experts Group. ISO/IEC JTC1/SC29/WG11. http://www . chiariglione.org/ mpeg/standards/mpeg-1/mpeg-1.htm.

[9] RealNetworks, Inc. RealAudio. http://www.realnetworks.com/products/codecs/ realaudio.html.

[10] RealNetworks, Inc. RealVideo. http://www.realnetworks.com/products/codecs/ realvideo.html.

[11] Peg Tyre. Professor in Your Pocket. Newsweek, pages 46-47, November 28, 2005. 